\title{
Group 3 LEA Protein, ZmLEA3, Is Involved in Protection from Low Temperature Stress
}

\author{
Yang Liu' ${ }^{1 *}$ Jianan Liang ${ }^{1}$, Liping Sun ${ }^{2}$, Xinghong Yang ${ }^{1}$ and Dequan Li ${ }^{1}$ \\ 1 State Key Laboratory of Crop Biology, Shandong Key Laboratory of Crop Biology, College of Life Sciences, Shandong \\ Agricultural University, Tai'an, China, ${ }^{2}$ Faculty of Chemistry and Chemical Engineering, Taishan Medical University, Tai'an, \\ China
}

Late embryogenesis abundant (LEA) proteins are a family of small highly hydrophilic proteins that accumulate at the onset of seed desiccation and in response to adverse conditions such as drought, salinity, low temperature, or water deficit. In previous studies, we demonstrated that ZmLEA3 could enhance the transgenic tobacco tolerance to osmotic and oxidative stresses. Here, we demonstrated that the transcription of $Z m L E A 3$ in the maize stems could be significantly induced by low temperature and osmotic stresses and by treatment with abscisic acid (ABA)

OPEN ACCESS

Edited by:

Shabir Hussain Wani,

Sher-e-Kashmir University

of Agricultural Sciences

and Technology, India

Reviewed by:

Vinay Kumar,

Modern College of Arts,

Science and Commerce, India

Rohit Joshi,

International Center for Genetic

Engineering and Biotechnology, India

${ }^{*}$ Correspondence:

Yang Liu

liuy@sdau.edu.cn

Specialty section:

This article was submitted to Crop Science and Horticulture,

a section of the journal

Frontiers in Plant Science

Received: 11 March 2016

Accepted: 27 June 2016

Published: 14 July 2016

Citation:

Liu Y, Liang J, Sun L, Yang $X$ and Li D (2016) Group 3 LEA Protein,

ZmLEA3, Is Involved in Protection from Low Temperature Stress.

Front. Plant Sci. 7:1011. doi: 10.3389/fpls.2016.01011 and $\mathrm{H}_{2} \mathrm{O}_{2}$. Further study indicated that $\mathrm{ZmLEA3}$ is a single copy gene in the maize genome. The $Z m L E A 3$ protein could protect lactate dehydrogenase $(\mathrm{LDH})$ activity at low temperatures. The overexpression of $Z m L E A 3$ conferred tolerance to low-temperature stress to transgenic tobacco, yeast (GS115) and E. coli (BL21).

Keywords: LEA protein, low-temperature stress, maize, ZmLEA3, transgenic gene

\section{INTRODUCTION}

Low-temperature damage is the main factor limiting plant growth and crop production. Plants have developed a wide variety of mechanisms to cope with environmental stresses. Accumulation of the late embryogenesis abundant (LEA) proteins is an important response to adverse conditions. LEA proteins accumulate in abundance during the late development stage of seeds (Dure et al., 1989; Tunnacliffe and Wise, 2007; Kosová et al., 2014; Chiappetta et al., 2015). Although the mechanistic basis for such protection is unclear, several studies have demonstrated that LEA proteins may protect cells during desiccation and freezing by acting as hydration buffers, sequestering ions and stabilizing proteins, membranes and chromatin structures (Amara et al., 2013; Battaglia and Covarrubias, 2013; Yang et al., 2015).

The LEA proteins can be classified into seven groups, in which the group 3 LEA proteins are characterized by a repeating motif of 11 amino acids (TAQAAKEKAGE; Dure, 1993). Because the number of repetitions of the 11-mer motif is different, the molecular mass in this group of proteins varies (Battaglia et al., 2008). Bioinformatics analysis indicated that the 11-mer exists principally as an amphipathic $\alpha$-helix. In previous studies, we have demonstrated that ZmLEA3 could enhance transgenic tobacco tolerance to osmotic and oxidative stresses. Although the functions of the group 3 LEA proteins remain unknown, they are assumed to be important for the establishment of environmental stress tolerance in seeds. Overexpressing wheat TaLEA3 and TaLEA2 in yeast improved transgenic yeast tolerance to hyperosmotic, salt, and freezing stresses (Yu et al., 2005). The synthetic peptide PvLEA-22 has the ability to preserve liposomes in the dry state (Furuki and Sakurai, 2014). 
Soybean group 3 LEA protein PM2 (seed maturation protein) conferred the tolerance to the $E$. coli recombinant against diverse stresses by protecting proteins and enzyme activity under lowor high-temperature conditions, and the 22-mer repeat region is an important functional region (Liu and Zheng, 2005; Liu et al., 2010).

LEA proteins were first found in cotton seeds and accumulated to high levels during the last stage of seed maturation and in water deficits in vegetative plant tissues (Dure et al., 1981). Many studies have reported that LEA proteins can be involved in abiotic and biotic stresses. Although some mechanisms have been proposed, the functional mechanism remains unclear (Brini et al., 2011; Eriksson et al., 2011; Petersen et al., 2012; Salleh et al., 2012). Previously, we demonstrated that the overexpression of $\mathrm{ZmLEA3}$ in tobacco resulted in improved osmotic stress tolerance and enhanced tolerance for oxidation stress (Liu et al., 2013). In the present study, we showed that the ZmLEA3 protein could protect enzymes from damage caused by low temperature. Further study indicated that the overexpression of ZmLEA3 conferred low-temperature stress tolerance in transgenic lines.

\section{MATERIALS AND METHODS}

\section{Plant Materials and Growth Condition}

The tobacco plants (Nicotiana tabacum cv NC 89) was used in this study. The tobacco seeds were plated on Murashige and Skoog (MS) medium (Murashige and Skoog, 1962) containing $200 \mathrm{mg} \mathrm{L}^{-1}$ kanamycin under light/dark cycle conditions of $16 / 8 \mathrm{~h}$ at $25^{\circ} \mathrm{C}$. For the low-temperature treatment, 6 -week-old transgenic tobacco lines were treated at $4^{\circ} \mathrm{C}$ under the same light periods. Samples were collected at the indicated time after the treatments, and all samples were frozen in liquid nitrogen immediately after collection and stored at $-80^{\circ} \mathrm{C}$.

\section{Bioinformatic Analysis}

Sequence identities were determined using BLAST on the NCBI web server ${ }^{1}$ and MaizeGDB ${ }^{2}$. The subcellular localizations were determined by means of the ProtComp v. 9.0 ${ }^{3}$. The isoelectric point ( $\mathrm{pI}$ ), molecular weight (MW), and grand average hydropathy (GRAVY) values were estimated by the ProtParam tool $^{4}$.

\section{Expression Profiles of ZmLEA3 in Stem under Different Stress Treatments Using Real-Time PCR}

Two-week-old maize was treated with $100 \mu \mathrm{M}$ abscisic acid (ABA), 20\% polyethylene glycol (PEG) 6000 (w/v), $20 \mu \mathrm{M} \mathrm{H}_{2} \mathrm{O}_{2}$, and low temperature $\left(4^{\circ} \mathrm{C}\right)$. Stem tissues collected from the

\footnotetext{
${ }^{1}$ http://blast.ncbi.nlm.nih.gov/Blast.cgi

${ }^{2}$ http://www.maizegdb.org/popcorn/search/sequence_search/home.php?a= BLAST_UI

${ }^{3}$ http://linux1.softberry.com/berry.phtml?topic=protcomppl\&group= Programs\&subgroup $=$ proloc

${ }^{4}$ http://us.expasy.org/tools/protparam.html
}

various treated plants at specific time points were immediately frozen in liquid nitrogen and stored at $-80^{\circ} \mathrm{C}$. The plants treated with only water were used as the experimental controls.

\section{Southern Blot Hybridizations}

Ten micrograms of genomic DNA from maize was digested by EcoRI, EcoRV, or BamHI restriction endonucleases. The restricted genomic DNA was separated on a $1.0 \%$ agarose gel and transferred to nylon membranes (Hybond- $\mathrm{N}^{+}$, Amersham) by capillary method using $20 \times$ saline sodium citrate (SSC). The restricted DNA was subsequently immobilized on the membrane by baking at $120^{\circ} \mathrm{C}$ for $30 \mathrm{~min}$. The coding sequence of the ZmLEA3 gene was used as a probe, which was labeled with $\left[\alpha-{ }^{32} \mathrm{P}\right] \mathrm{dATP}$ using a random-primed method.

\section{In Vitro Stress Assays and LDH Activity Measurements}

Lactate dehydrogenase (LDH) (Roche, UK) from rabbit muscle was diluted in $25 \mathrm{mM}$ Tris- $\mathrm{HCl}, \mathrm{pH}$ 7.5. The low-temperature treatment was assayed according to the previously published method (Reyes et al., 2008). The mixture in a final volume of $75 \mu \mathrm{l}$ was frozen for $15 \mathrm{~min}$ in dry ice and thawed for $15 \mathrm{~min}$ in a water bath at $25^{\circ} \mathrm{C}$. The final enzyme concentration was $200 \mathrm{nM}$. For LDH activity, the assay buffer was $25 \mathrm{mM}$ Tris- $\mathrm{HCl} \mathrm{pH} 7.5$ containing $2 \mathrm{mM}$ pyruvate (Sigma) and $0.15 \mathrm{mM} \mathrm{NADH}$ (dihydronicotinamide-adenine dinucleotide, Roche). LDH activity was monitored as the rate of decrease in absorbance at $340 \mathrm{~nm}$ for $1 \mathrm{~min}$ due to the conversion of $\mathrm{NADH}$ into $\mathrm{NAD}$ at $25^{\circ} \mathrm{C}$. The rate determined for the untreated samples was considered to be $100 \%$ in all graphs.

\section{Expression of ZmLEA3 in Pichia Yeast GS115 and E. coli BL21}

The transgenic yeast (Ppic3.5K-ZmLEA3) and E. coli (PET30ZmLEA3) were kept in our labs. The $Z m L E A 3$ gene was inserted into the pET30 vector (pET system) and then transformed into E. coli BL21 Star cells to create the BL/ZmLEA3 recombinant. The expression of recombinant proteins with $6 \times$ His tag at $\mathrm{N}$-terminus was performed following the manufacturer's protocol (pET system). The Pichia GS115 strain was transformed with $5 \mu \mathrm{g}$ of linearized plasmid by the $\mathrm{LiCl}$ method according to the EasySelect Pichia Expression Kit (Invitrogen, USA).

\section{Low-Temperature Tolerance Assays of Yeast and E. coli Transformants}

Low-temperature tolerance assays were performed as described previously (Liu et al., 2014). For yeast, the recombinant yeast was inoculated in $25 \mathrm{ml}$ BMGY (buffered glycerol-complex medium, $1 \%$ yeast extract, $2 \%$ peptone, $1.34 \%$ yeast nitrogen base (YNB), $10 \mathrm{mM} \mathrm{K}_{3} \mathrm{PO}_{4}, 4 \times 10^{-5} \mathrm{mM}$ biotin, and $1 \%$ glycerin). After $18 \mathrm{~h}$ of incubation at $28^{\circ} \mathrm{C}$, the cells were collected by centrifugation and resuspended in $200 \mathrm{ml}$ induction BMMY (buffered methanol-complex medium, $1 \%$ yeast extract, $2 \%$ peptone, $10 \mathrm{mM} \mathrm{K}_{3} \mathrm{PO}_{4}, 1.34 \% \mathrm{YNB}, 4 \times 10^{-5} \mathrm{mM}$ biotin, and $0.5 \%$ methanol) and then incubated at $28^{\circ} \mathrm{C}$ for 4 days. 
TABLE 1 | Characteristics of the group 3 LEA genes identified in maize.

\begin{tabular}{|c|c|c|c|c|c|c|c|c|}
\hline Name & Accession no. & Chromosome localization & Length & MW & GRAVY & $\mathrm{pl}$ & Intron & Location \\
\hline ZmLEA3-1 & NM_001153473.1 & $6: 161966271-161967560$ & 182 & 18599 & -1.102 & 7.84 & 2 & Mitochondrial or nuclear \\
\hline ZmLEA3-2 & ВT062068.2 & 10:117570524-117572326 & 354 & 37909 & -1.036 & 6.6 & 1 & Cytoplasmic \\
\hline ZmLEA3-3 & NM_001111828.1 & $9: 133421515-133422685$ & 221 & 22759 & -1.204 & 8.8 & 1 & Cytoplasmic and nuclear \\
\hline ZmLEA3-4 & NM_001156540.1 & 6:161966444-161966664 & 320 & 33572 & -0.932 & 6.16 & 0 & Plasma membrane \\
\hline ZmLEA3-5 & NM_001156540.1 & $1: 14450012-14450363$ & 305 & 32278 & -0.887 & 8.39 & 0 & Nuclear \\
\hline ZmLEA3-6 & EU952743.1 & 2:10718061-10178389 & 205 & 21190 & -0.796 & 8.98 & 0 & Cytoplasmic \\
\hline ZmLEA3-7 & AFW71082.1 & $5: 142403718-142404354$ & 604 & 61763 & -0.814 & 7.29 & 0 & Chloroplast membrane \\
\hline
\end{tabular}

A

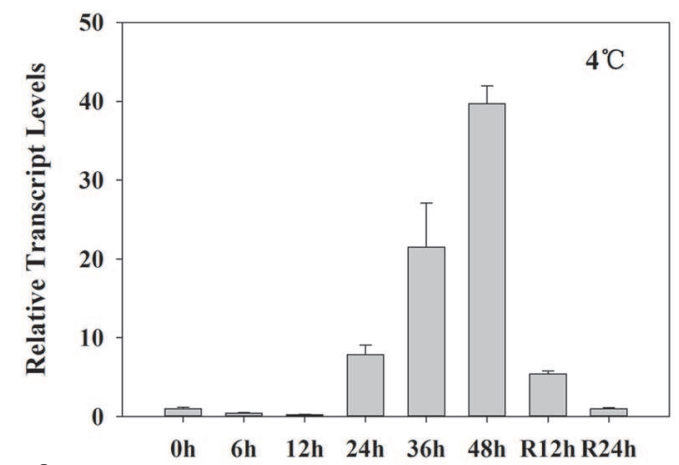

C
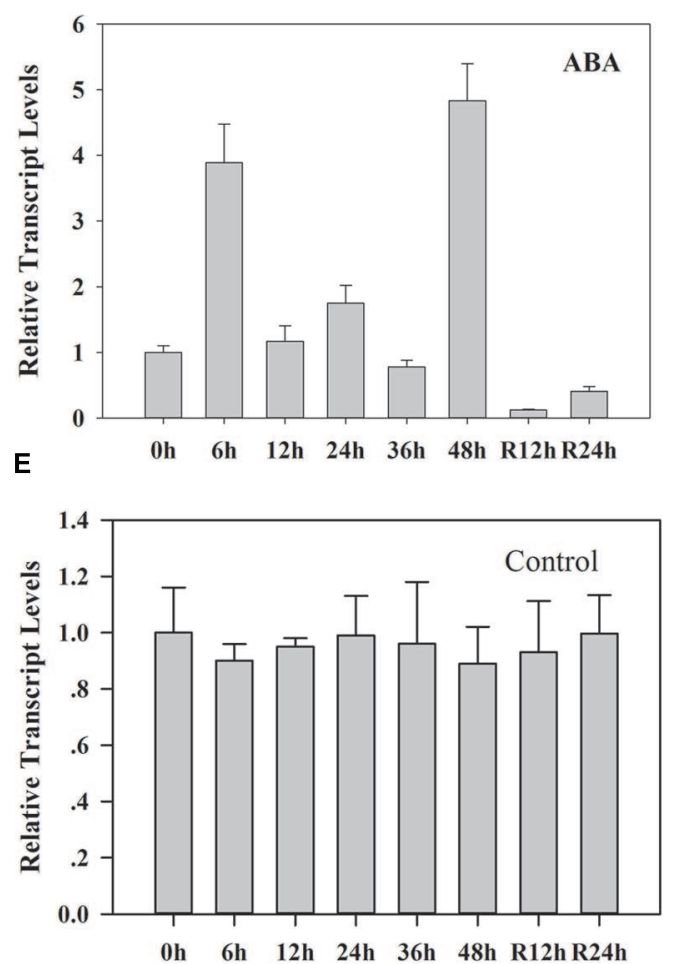

B
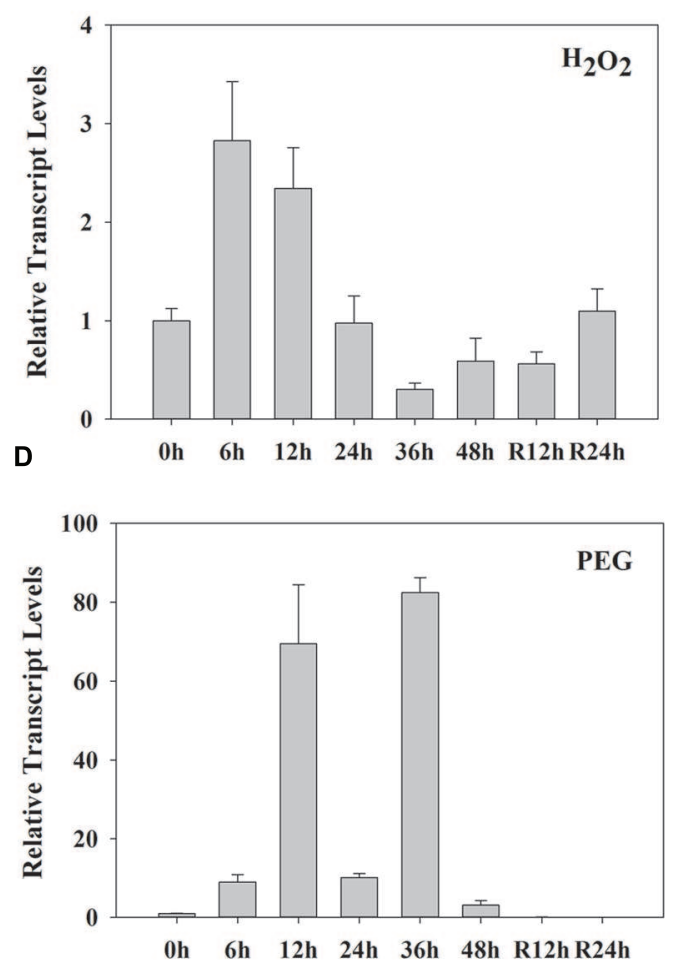

FIGURE 1 | Transcript accumulation of ZmLEA3 in maize stems. The maize seedlings were incubated with Hoagland's solution for 2 weeks; uniformly sized plants at similar growth stages were chosen for further study. The total RNA was isolated from the stems. Maize seedlings were treated with $4^{\circ} \mathrm{C}(\mathbf{A})$, $10 \mathrm{mM} \mathrm{H}_{2} \mathrm{O}_{2}$ (B), $100 \mu \mathrm{M}$ abscisic acid (ABA) (C), 20\% polyethylene glycol (PEG) 6000 (w/v) (D), or water (control) (E). Total RNA was isolated from the stems at the indicated times after the treatments. Each curve/column represents an average of three replicates, and error bars represent standard deviation. $\mathrm{R}$ represents removal of the treatment. 
Methanol was added every $24 \mathrm{~h}$ to a final concentration of $0.5 \%$. To assay the low-temperature tolerance of the E. coli recombinant, the cultures were induced with isopropyl $\beta$-D-1thiogalactopyranoside (IPTG, $1 \mathrm{mM}$ ) for $4 \mathrm{~h}$.

For low-temperature treatment, the culture (yeast or E. coli, $1 \mathrm{ml}$ ) was cooled at $-20^{\circ} \mathrm{C}$ for $24 \mathrm{~h}$ and then inoculated into $150 \mathrm{ml}$ of BMGY or lysogeny broth (LB) medium, respectively. At each time point, $3 \mathrm{ml}$ of culture was used to measure the $\mathrm{OD}_{600}$ with a spectrophotometer.

\section{Overexpression of ZmLEA3 in Tobacco}

The coding regions of $Z m L E A 3$ were ligated into the binary pBI121 expression vector under the CaMV 35S (promoter of cauliflower mosaic virus) promoter. Constructs were transformed into Agrobacterium tumefaciens strain LBA4404 and then transformed into tobacco plants by using the leaf disc transformation method.

\section{Germination Experiments}

Approximately 50 surface-sterilized seeds from each homozygous T2 transgenic line and the wild-type (WT) tobacco line were plated on solid media composed of MS basal salts and 0.8\% sucrose. To determine cold-stress sensitivity, the plates were incubated in a controlled-environment growth chamber at $12^{\circ} \mathrm{C}$ with a photoperiod of $16 / 8 \mathrm{~h}$ (day/night). Thirty days later, the rates of seed germination were evaluated (root emergence). Each experiment was repeated a minimum of three times with identical results.

\section{Low-Temperature Stress Treatments and Assays in Plants}

Six-week-old tobacco seedlings were kept at $4^{\circ} \mathrm{C}$ for the indicated time, and then $0.5 \mathrm{~g}$ of leaves was collected for malondialdehyde (MDA) and relative electrolytic leakage measurements, which were performed as described previously (Liu et al., 2013; Wang et al., 2014). The activity of superoxide dismutase (SOD) and peroxidase (POD) measurements that were performed as described previously (Jiang and Zhang, 2001).

\section{Statistical Analysis}

Statistical analyses and plotting were performed using SigmaPlot and SPSS software. The statistical significance of the difference was confirmed by Student's $t$-test, ${ }^{*} P<0.05$; ${ }^{* *} P<0.01$.

\section{RESULTS AND DISCUSSION}

\section{The Group 3 LEA Proteins Encoding Genes in the Maize Genome}

The LEA protein family is very large in plants, with up to 51 genes encoding LEA proteins in Arabidopsis thaliana (Hundertmark and Hincha, 2008). Using the group 3 LEA protein genes from $A$. thaliana, we performed BLAST searches against the maize genomic database. According to the results, this analysis identified seven putative group 3 LEA protein genes in the complete maize genome (Table 1). All of these proteins were

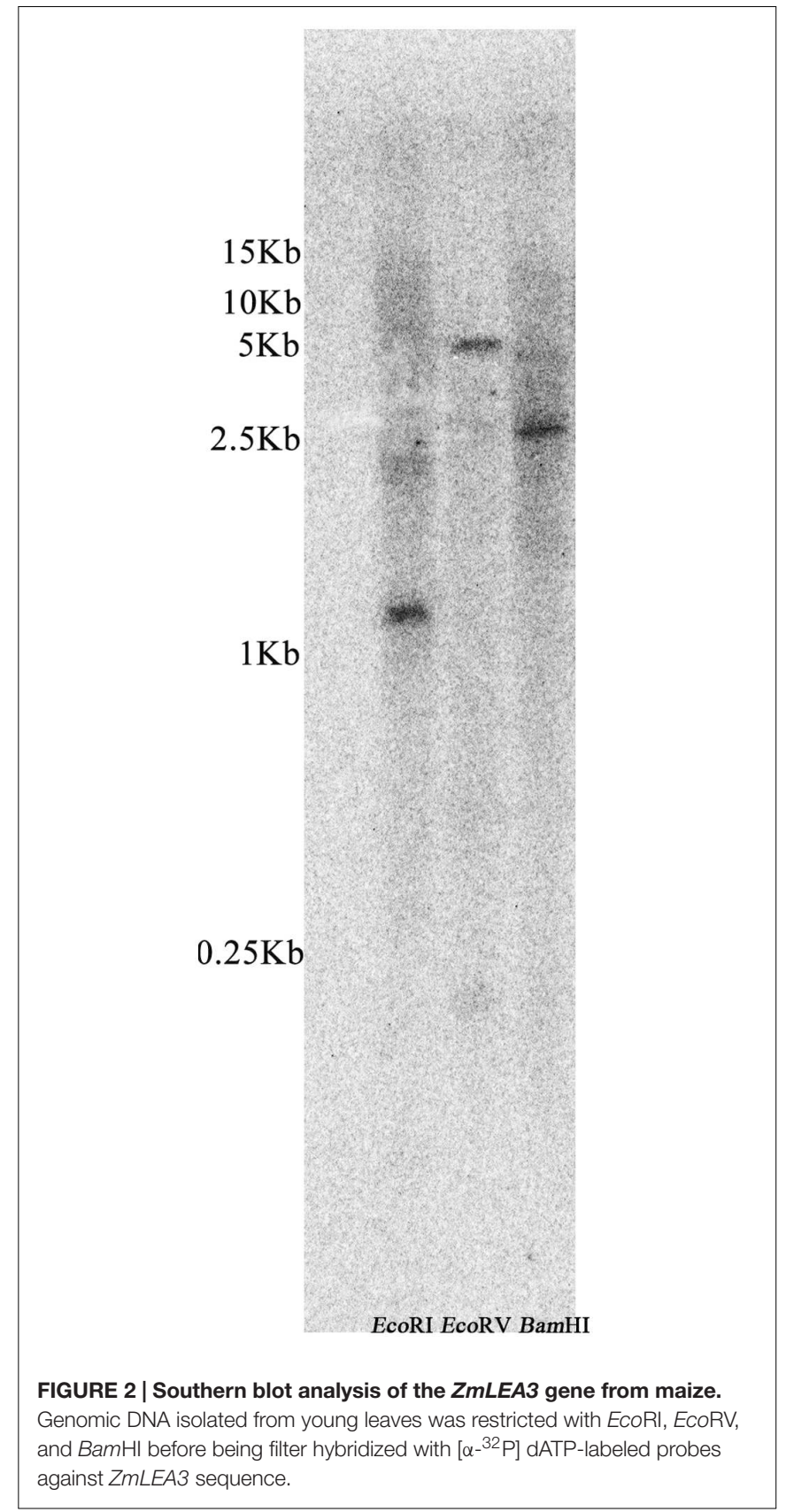

highly hydrophobic with grand average hydrophobicities from -1.024 to -0.796 and theoretical $\mathrm{p} I$ values from 6.16 to 8.98 . The MWs of these proteins ranged from 18.6 to $61.8 \mathrm{kDa}$. We also predicted the subcellular localizations by means of ProtComp v.9.0. The results indicated that the group 3 LEA proteins were localized in the nucleus, cytoplasm, plasma membrane, and chloroplast membrane.

\section{Accumulation of the ZmLEA3 Transcript under Different Stress Treatments}

A previous study has demonstrated that the transcription of $Z m L E A 3$ was higher in the stems than in the roots and leaves 
A

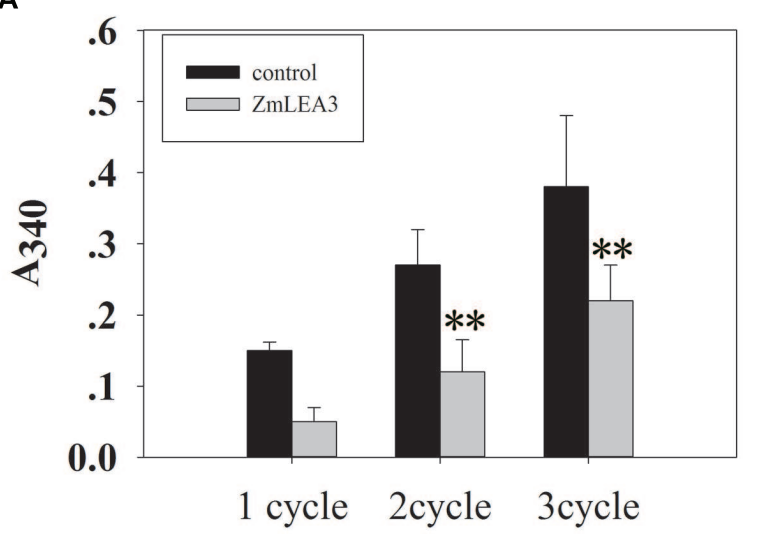

B

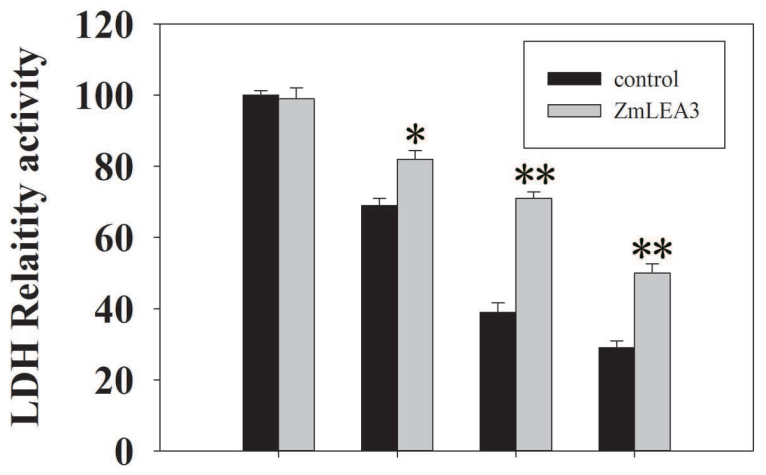

0cycle 1cycle 2cycle 3cycle

FIGURE 3 | The ZmLEA3 protein could protect lactate dehydrogenase (LDH) from damage caused by low-temperature stress. LDH (Roche, UK) from rabbit muscle was diluted in $25 \mathrm{mM}$ Tris- $\mathrm{HCl}$, pH 7.5. (A) Aggregation of $\mathrm{LDH}$ after low-temperature stress in the presence of $0.24 \mathrm{mg}$ of $\mathrm{ZmLEA3}$ or bovine serum albumin (BSA). Aggregation was measured by the effect of light scattering giving an apparent $A_{340}$ in the spectrophotometer. (B) The effect of low temperature on LDH activity. Each curve/column represents an average of three replicates, and error bars represent standard deviation. The data of the control plant and the transgenic plant were compared for the significance difference at each respective time point, ${ }^{*} P<0.05,{ }^{* *} P<0.01$.

A

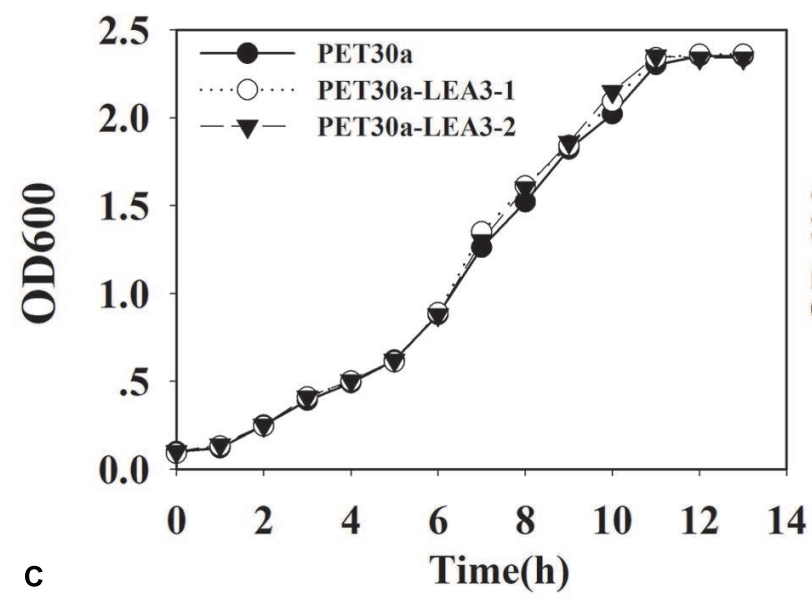

c

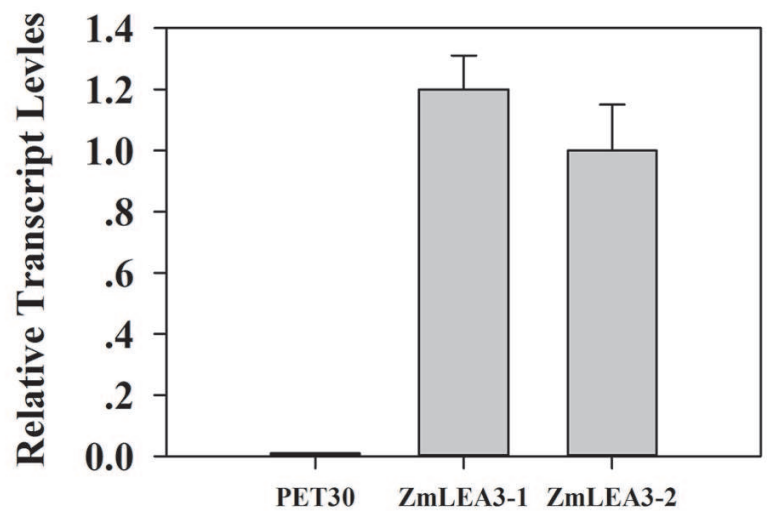

B

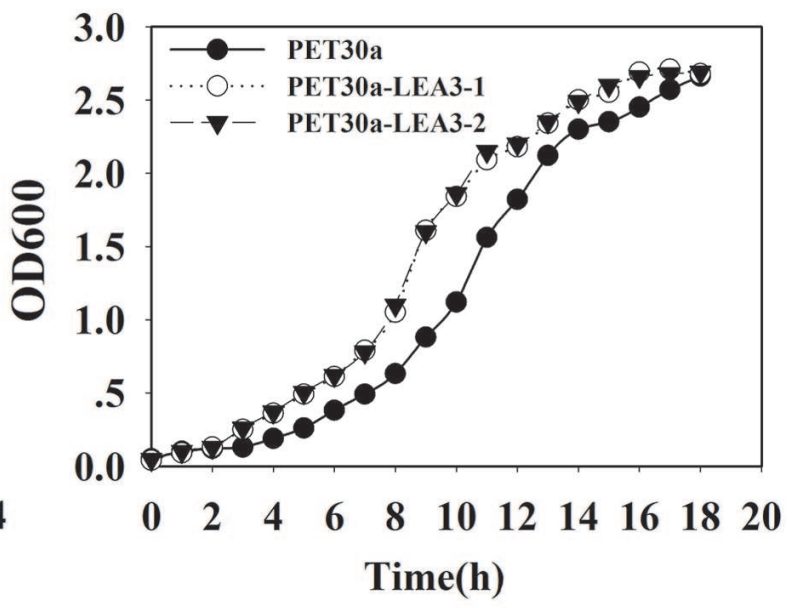

FIGURE 4 | Overexpression enhances tolerance to low-temperature stress in the E. coli ZmLEA3 transformant. (A) The transformant E. coli (BL21) with the empty vector or PET30-ZmLEA3 were grown in lysogeny broth (LB) medium. (B) The transformant E. coli (BL21) were grown in the non-stress LB medium after $-20^{\circ} \mathrm{C}$ treatment. Two yeast and E. coli transformants were chosen for further study. (C) Accumulation of the $Z m L E A 3$ in transgenic E. coli. Each curve/column represents an average of three replicates, and error bars represent standard deviation. 

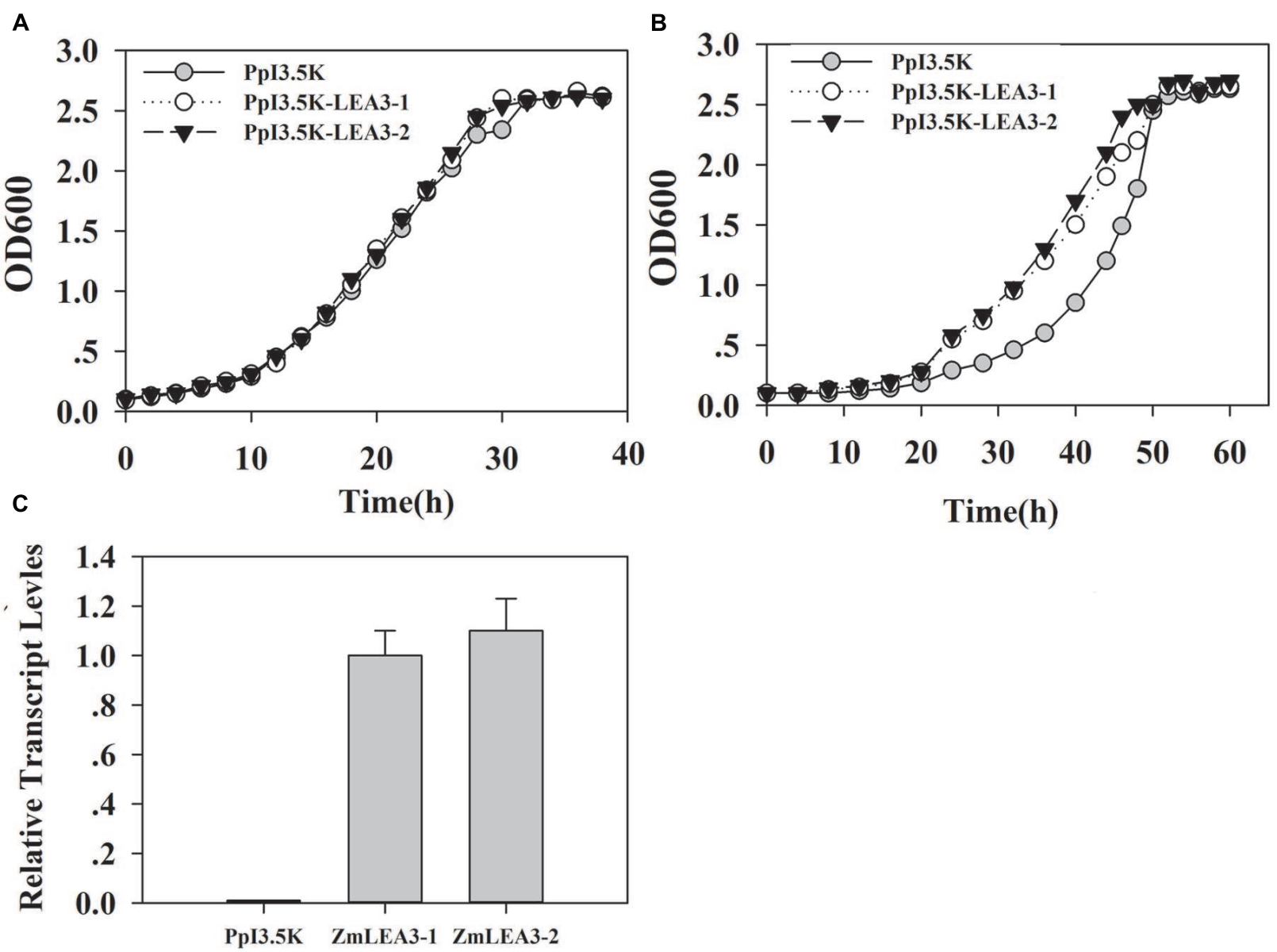

FIGURE 5 | Overexpression enhances tolerance to low-temperature stress in the yeast ZmLEA3 transformant. (A) The transformant yeasts (GS115) with the empty vector or Ppl3.5K-ZmLEA3 were grown in the non-stress buffered glycerol-complex medium (BMGY). (B) The transformant yeasts were grown in the non-stress BMGY medium after $-20^{\circ} \mathrm{C}$ treatment. (C) Accumulation of the $Z m L E A 3$ in transgenic yeasts. Each curve/column represents an average of three replicates, and error bars represent standard deviation.

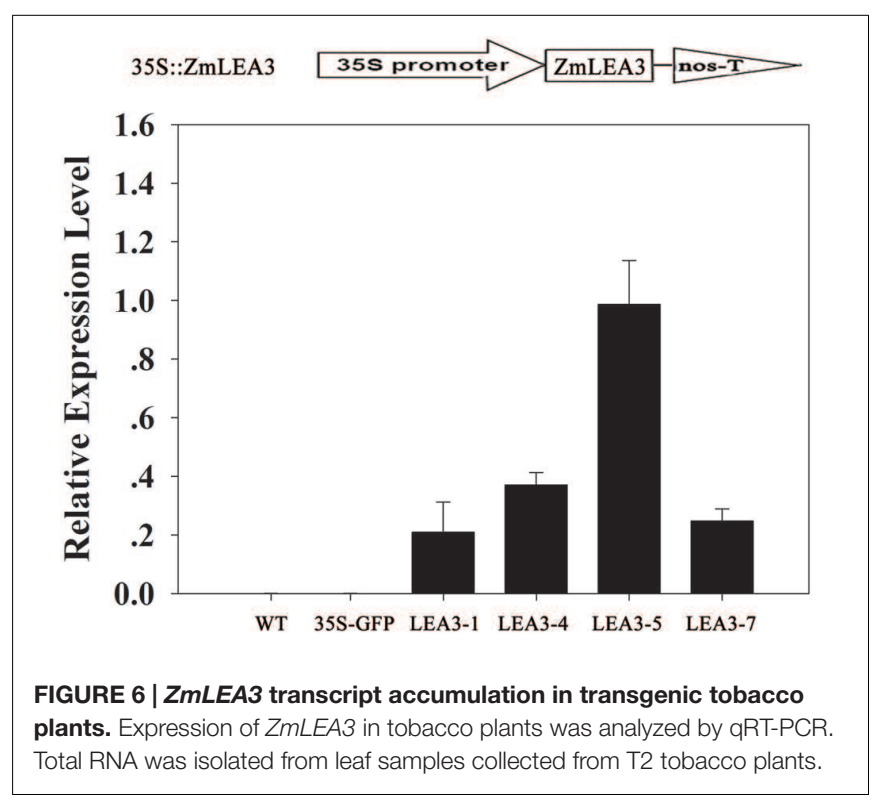

(Liu et al., 2013). To further determine the expression pattern of $Z m L E A 3$, quantitative real-time reverse transcription-PCR (qRT-PCR) was performed to examine the transcript levels of $Z m L E A 3$ in the maize stems. The results demonstrated that the transcript accumulation of ZmLEA3 could be induced by dehydration and low temperature as well as by treatment with $\mathrm{ABA}$ and $\mathrm{H}_{2} \mathrm{O}_{2}$ (Figure 1). In the low-temperature treatment, the transcript level of $Z m L E A 3$ reached its highest level suddenly at $48 \mathrm{~h}$ and then reverted back to near its control level immediately when the signal was removed. The transcript accumulation of $\mathrm{ZmLEA3}$ in response to $\mathrm{H}_{2} \mathrm{O}_{2}$ reached its highest level at $6 \mathrm{~h}$ and then was reduced to its usual normal level. The transcript level of $Z m L E A 3$ in response to ABA treatment reached a high peak within $6 \mathrm{~h}$ and then was reduced at $12 \mathrm{~h}$, but its transcript accumulation reached its highest level at $48 \mathrm{~h}$ and decreased to close to its uninduced level when the treatment was removed. For 20\% PEG treatment, there were two peaks, with the transcript accumulation reaching a high level at 12 and $36 \mathrm{~h}$, suggesting the existence of a feedback adjustment. 

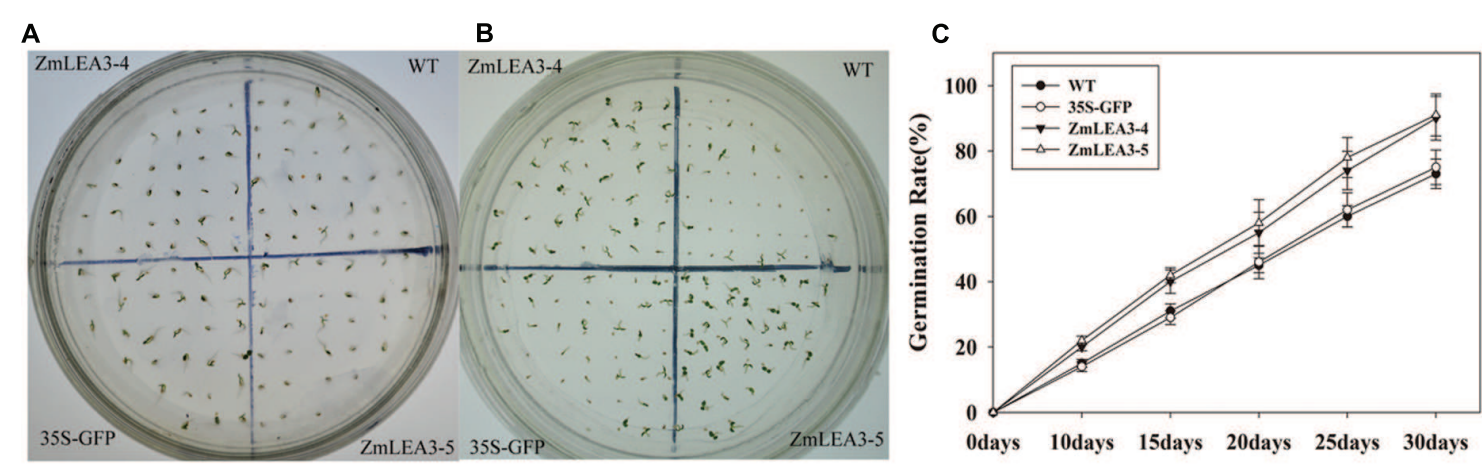

FIGURE 7 | The primary analysis of the low-temperature stress tolerance in transgenic tobacco plants. The phenotype of transgenic and control tobacco seeds grown for 4 weeks under normal condition $\mathbf{( A )}$ or low-temperature $\left(\mathbf{B}, 12^{\circ} \mathrm{C}\right)$ stress. (C) The seed germination rate of the tobacco seeds under low-temperature $\left(12^{\circ} \mathrm{C}\right)$ stress.

\section{ZmLEA3 is a Single Copy Gene in the Zea mays Genome}

Southern blot analysis was performed to investigate whether the homology gene of $Z m L E A 3$ was present in the $Z$. mays genome. As shown in Figure 2, only one hybridization band of the digested fragments was observed with the probe. This result indicated that ZmLEA3 is a single copy gene in the $Z$. mays genome.

\section{ZmLEA3 Protein Protected LDH Activity from Damage Caused by Low-Temperature Stress}

It has been previously demonstrated that freeze-thaw can reduce LDH activity (Reyes et al., 2008). To determine the antiaggregation activity of the ZmLEA3 protein, an assay was used to measure the protective effect of ZmLEA3 proteins on $\mathrm{LDH}$ activity. As shown in Figure 3, ZmLEA3 could protect the activity and reduce the aggregation of $\mathrm{LDH}$ under freeze-thaw cycle.

$\mathrm{LDH}$ as a reporter enzyme had been used to test the protection activity of different LEA proteins from different groups and species (Tunnacliffe and Wise, 2007). It has been hypothesized that LEA proteins may play protective roles for proteins by acting as chaperone molecules (Kovacs et al., 2008). In this report, we demonstrated that ZmLEA3 could protect the LDH activity from damage caused by freeze-thaw cycle. HernándezSánchez et al. (2014) reported that LEA proteins could function through the formation of dimers and large multimeric complexes, forming a large molecular shield around its biological targets. These results indicated that LEA proteins could prevent protein aggregation under environmental stresses by stabilizing protein species in a partially unfolded state. According to these results, we demonstrated that ZmLEA3 proteins could protect the activity of protein from damage caused by low temperature.

\section{Overexpression ZmLEA3 Conferred Tolerance to Low Temperature on Transgenic Yeast and E. coli}

To determine the function of the ZmLEA3 fusion protein in low-temperature stress, the effects of low temperature on the growth of the recombinant $E$. coli and yeast were examined under low-temperature stress. The growth curves of the yeast and E. coli cell lines transformed with the pPI3.5k-ZmLEA5C or PET30-ZmLEA3 vector and the control lines containing the empty vector (pPI3.5k or PET30) were measured under lowtemperature stress. Under optimal conditions, there was no significant difference between the transformed lines and the control. However, under low-temperature stress, the transformed yeast and E. coli displayed higher growth compared with the control, and the lag phase of the transformed yeasts and E. coli was shorter than that the control (Figures 4 and 5).

The phosphorylation and dephosphorylation of LEA protein seems to be a major post-translational modification (Rorat, 2006). The phosphorylation of maize dehydrin Rab17 by protein kinase CKII is the relevant step for its nuclear localization (Jensen et al., 1998). Jensen et al. (1998) reported that phosphorylation regulated ion-binding is a property shared by the acidic subclass dehydrins. Because recombinant protein is not phosphorylated by $E$. coli, the phosphorylation did not influence the function of ZmLEA3. Here, our results indicated that overexpression of ZmLEA3 could enhance the tolerance to low-temperature in transgenic yeast and E. coli. This result is consistent with a previous study, in which a group 3 LEA protein was not phosphorylated by protein kinase (Amara et al., 2012).

\section{Overexpression of ZmLEA3 Enhanced Transgenic Tobacco Tolerance to Low-Temperature Stress}

A previous study indicated that ZmLEA3 could enhance tolerance to drought and oxidative stresses in transgenic tobacco and yeast (Liu et al., 2013). To further address the function of the ZmLEA3 protein, the low-temperature tolerance of the transgenic tobacco was analyzed. Independent transgenic lines were obtained by kanamycin-resistance selection and confirmed by genomic PCR (data not shown). The expression levels of $Z m L E A 3$ in tobacco were detected by qRT-PCR, whereas no expression was detected in the control plants (WT and 35S-GFP/green fluorescent protein). Two (LEA3-4 and LEA3-5) of the ZmLEA3 transgenic 
A

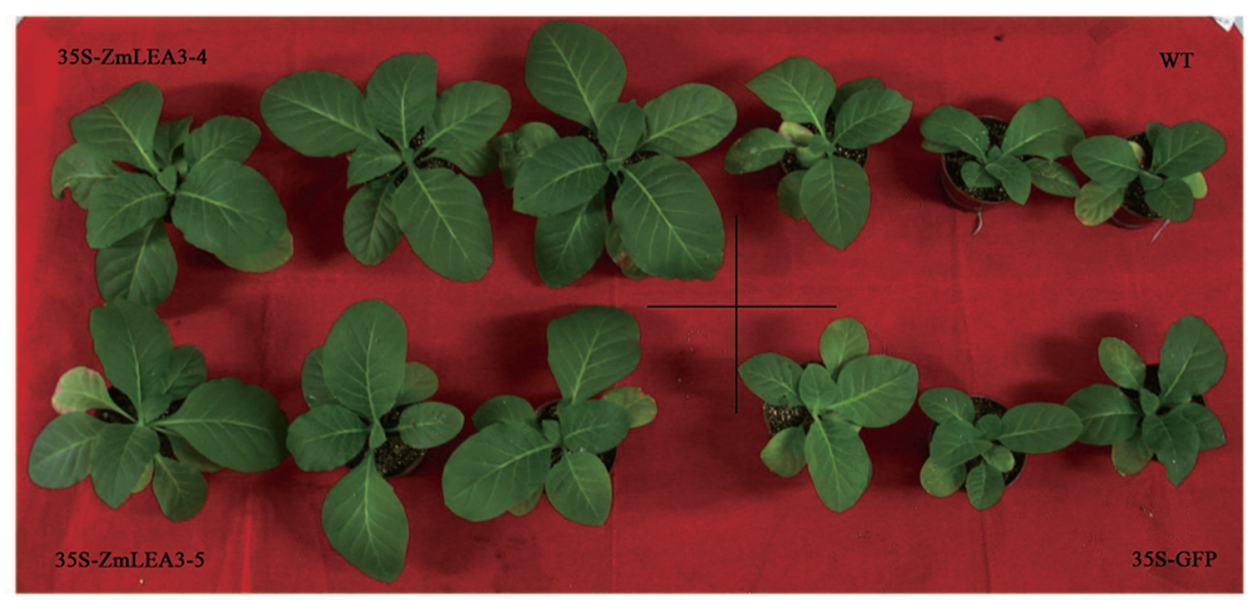

B

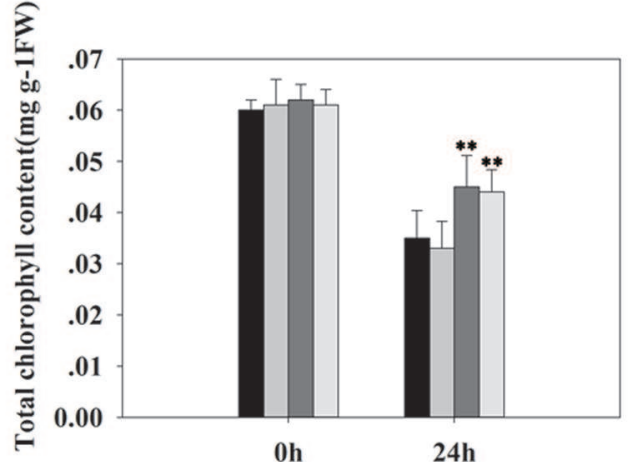

D
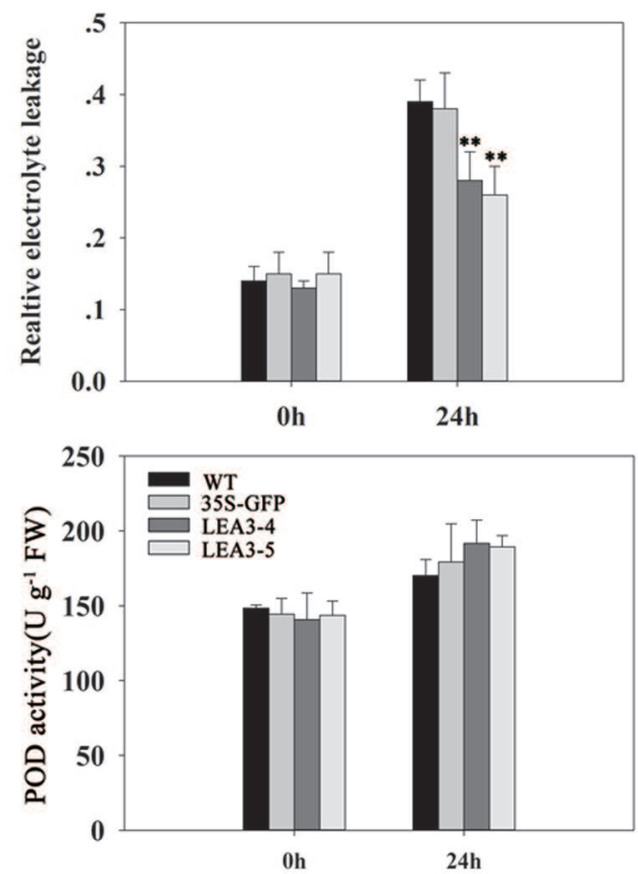

C

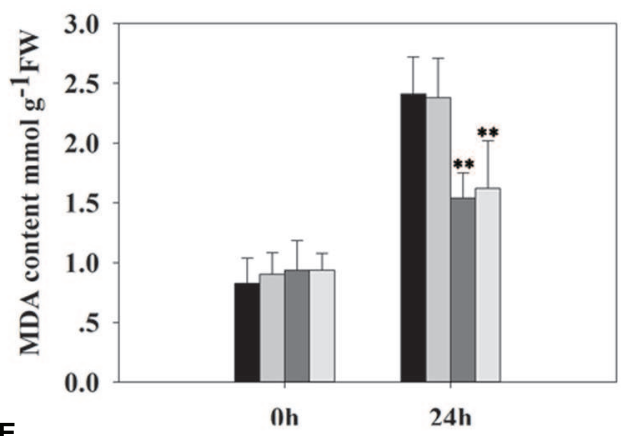

E

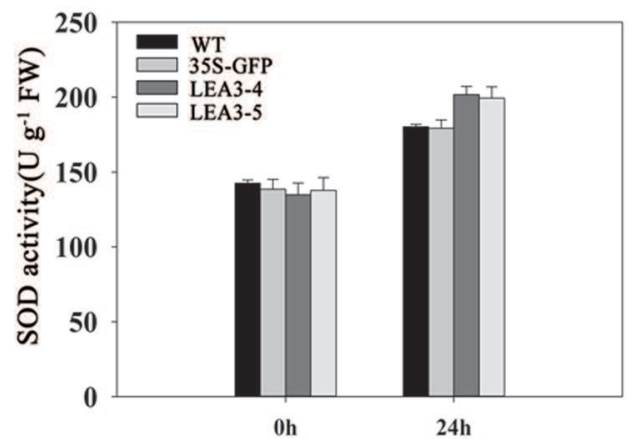

oh

24h

WT

35S-GFP

35S-ZmLEA3-4 35S-ZmLEA3-5

FIGURE 8 | The low-temperature tolerance of transgenic and control tobacco plants. The phenotype of transgenic and control tobacco plants grown in normal conditions after low-temperature treatment (A). Total chlorophyll content (B), malondialdehyde (MDA) (C), the relative electrolyte leakage (D), the activity of superoxide dismutase (SOD) (E), and peroxidase (POD) (F) were measured in 6-week-old transgenic and control tobacco plants before and after low-temperature treatment $\left(4^{\circ} \mathrm{C}\right)$ at the indicated time. Each curve/column represents an average of three replicates, and error bars represent standard deviation. The statistical significance of the difference was confirmed by Student's $t$-test, ${ }^{* *} P<0.01$. 
lines were chosen for further analysis (Figure 6). As shown in Figure $\mathbf{7 A}$, there were no differences in the germination rate between the transgenic lines and the control plants (WT and 35S-GFP) under the control conditions. However, the germination rate of the transgenic tobacco plants was higher than that of the control plants under low-temperature stress (Figures 7B,C).

Several reports have demonstrated that LEA proteins enhance transgenic plants low-temperature tolerance, but there is little known about the roles of the maize group 3 LEA proteins under low-temperature stress. Yu et al. (2005) reported that overexpression of the group 3 LEA proteins TaLEA2 and TaLEA3 improved transgenic yeast cell tolerance to lowtemperature stress, and yeast transformants with TaLEA2 seemed to be more tolerant to freezing stress than transformants with TaLEA3. Overexpression of the barley HVA1 proteins in Saccharomyces cerevisiae resulted in the generation of freezingtolerant organisms (Zhang et al., 2000). To investigate the low-temperature tolerance of the transgenic plants, the tobacco plants were treated at $4^{\circ} \mathrm{C}$ for $24 \mathrm{~h}$ and were then transferred to normal conditions. As shown in Figure $\mathbf{8 A}$ that the transgenic plants grew significantly better than the control plants. Chlorophyll content can be used to estimate the degree of leaf senescence. Under normal conditions, there is no significant difference in the chlorophyll levels, but the chlorophyll content was also significantly reduced in the control plants compared with the transgenic plants (Figure 8B). MDA content and the relative electrolyte leakage can represent the membrane injury. In this study, our results indicated that cold stress significantly increased MDA content and relative electrolyte leakage in the control plants compared with the transgenic plants (Figures 8C,D).

Antioxidant enzymes scavenge reactive oxygen species (ROS), which can accumulate when stress is imposed. Based on the

\section{REFERENCES}

Amara, I., Capellades, M., Ludevid, M. D., Pagès, M., and Goday, A. (2013). Enhanced water stress tolerance of transgenic maize plants over-expressing LEA Rab28 gene. J. Plant Physiol. 170, 864-873. doi: 10.1016/j.jplph.2013.01.004

Amara, I., Odena, A., Oliveira, E., Moreno, A., Masmoudi, K., Pagés, M., et al. (2012). Insights into maize LEA proteins: from proteomics to functional approaches. Plant Cell Physiol. 53, 312-329. doi: 10.1093/pcp/pcr183

Battaglia, M., and Covarrubias, A. A. (2013). Late embryogenesis abundant (LEA) proteins in legumes. Front. Plant Sci. 4:190. doi: 10.3389/fpls.2013.00190

Battaglia, M., Olvera-Carrillo, Y., Garciarrubio, A., Campos, F., and Covarrubias, A. A. (2008). The enigmatic LEA proteins and other hydrophilins. Plant Physiol. 148, 6-24. doi: 10.1104/pp.108.120725

Brini, F., Yamamoto, A., Jlaiel, L., Takeda, S., Hobo, T., Dinh, H. Q., et al. (2011). Pleiotropic effects of the wheat dehydrin DHN-5 on stress responses in Arabidopsis. Plant Cell Physiol. 52, 676-688. doi: 10.1093/pcp/pcr030

Chiappetta, A., Muto, A., Bruno, L., Woloszynska, M., Van Lijsebettens, M., and Bitonti, M. B. (2015). A dehydrin gene isolated from feral olive enhances drought tolerance in Arabidopsis transgenic plants. Front. Plant Sci. 6:392. doi: $10.3389 /$ fpls.2015.00392

Dure, L. (1993). A repeating 11-mer amino acid motif and plant desiccation. Plant J. 3, 363-369. doi: 10.1046/j.1365-313X.1993.t01-19-00999.X

Dure, L., Crouch, M., Harada, J., Ho, T. H. D., Mundy, J., Quatrano, R., et al. (1989). Common amino acid sequence domains among the LEA proteins of higher plants. Plant Mol. Biol. 12, 475-486. doi: 10.1007/BF00036962 above results that the ZmLEA3 protein could protect the activity of LDH in low-temperature stress, we speculated that ZmLEA3 could protect antioxidant enzymes such as SOD or POD. To confirm this hypothesis, we checked the activity of SOD and POD. As shown in Figures 8E,F, the activity of SOD and POD was higher in the transgenic lines than in the control plants after low-temperature treatment.

\section{CONCLUSION}

In summary, these findings provide evidence that ZmLEA3 is a single copy gene in the $Z$. mays genome and plays an important role in conferring low-temperature tolerance in prokaryotes and eukaryotes. We propose that the group 3 LEA protein may serve as a membrane and protein stabilizer during low-temperature stress. However, the protective role has not been completely elucidated, and further experiments are necessary to explore the molecular mechanism.

\section{AUTHOR CONTRIBUTIONS}

YL and DL designed the experiments. YL performed the experiments and analyzed the data. YL and JL wrote the article. LS and XY gave positive suggestion about this article. All authors read and approved the manuscript.

\section{ACKNOWLEDGMENT}

This work was supported by the grants from the National Natural Science Foundation of China (Nos. 31071337 and 31271633) and the Taishan Medical University (2015GCC20).

Dure, L., Greenway, S. C., and Galau, G. A. (1981). Developmental biochemistry of cottonseed embryogenesis and germination: changing messenger ribonucleic acid populations as shown by in vitro and in vivo protein synthesis. Biochemistry 20, 4162-4168. doi: 10.1021/bi00517a033

Eriksson, S. K., Kutzer, M., Procek, J., Grobner, G., and Harryson, P. (2011). Tunable membrane binding of the intrinsically disordered dehydrin Lti30, a cold-induced plant stress protein. Plant Cell 23, 2391-2404. doi: $10.1105 /$ tpc. 111.085183

Furuki, T., and Sakurai, M. (2014). Group 3 LEA protein model peptides protect liposomes during desiccation. Biochim. Biophys. Acta 1838, 2757-2766. doi: 10.1016/j.bbamem.2014.07.009

Hernández-Sánchez, I. E., Martynowicz, D. M., Rodríguez-Hernández, A. A., Pérez-Morales, M. B., Graether, S. P., and Jiménez-Bremont, J. F. (2014). A dehydrin-dehydrin interaction: the case of SK3 from Opuntia streptacantha. Front. Plant Sci. 5:520. doi: 10.3389/fpls.2014. 00520

Hundertmark, M., and Hincha, D. K. (2008). LEA (late embryogenesis abundant) proteins and their encoding genes in Arabidopsis thaliana. BMC Genomics 9:118. doi: 10.1186/1471-2164-9-118

Jensen, A. B., Goday, A., Figueras, M., and Jessop, A. C. (1998). Phosphorylation mediates the nuclear targeting of the maize Rab17 protein. Plant J. 13, 691-697. doi: 10.1046/j.1365-313X.1998.00069.x

Jiang, M., and Zhang, J. (2001). Effect of abscisic acid on active oxygen species, antioxidative defence system and oxidative damage in leaves of maize seedlings. Plant Cell Physiol. 42, 1265-1273. doi: 10.1093/pcp/pce162 
Kosová, K., Vítámvás, P., and Prášil, I. T. (2014). Wheat and barley dehydrins under cold, drought, and salinity-what can LEA-II proteins tell us about plant stress response? Front. Plant Sci. 5:343. doi: 10.3389/fpls.2014. 00343

Kovacs, D., Kalmar, E., Torok, Z., and Tompa, P. (2008). Chaperone activity of ERD10 and ERD14, two disordered stress-related plant proteins. Plant Physiol. 147, 381-390. doi: 10.1104/pp.108.118208

Liu, Y., Wang, L., Jiang, S. S., Pan, J. W., Cai, G. H., and Li, D. Q. (2014). Group 5 LEA protein, ZmLEA5C, enhance tolerance to osmotic and low temperature stresses in transgenic tobacco and yeast. Plant Physiol. Biochem. 84, 22-31. doi: 10.1016/j.plaphy.2014.08.016

Liu, Y., Wang, L., Xing, X., Sun, L., Pan, J., Kong, X., et al. (2013). ZmLEA3, a multifunctional group 3 LEA protein from maize (Zea mays L.), is involved in biotic and abiotic stresses. Plant Cell Physiol. 54, 944-959. doi: $10.1093 / \mathrm{pcp} / \mathrm{pct} 047$

Liu, Y., and Zheng, Y. (2005). PM2, a group 3 LEA protein from soybean, and its 22-mer repeating region confer salt tolerance in Escherichia coli. Biochem. Biophys. Res. Commun. 331, 325-332. doi: 10.1016/j.bbrc.2005.03.165

Liu, Y., Zheng, Y., Zhang, Y., Wang, W., and Li, R. (2010). Soybean PM2 protein (LEA3) confers the tolerance of Escherichia coli and stabilization of enzyme activity under diverse stresses. Curr. Microbiol. 60, 373-378. doi: 10.1007/s00284-009-9552-2

Murashige, T., and Skoog, F. (1962). A revised medium for rapid growth and bioassay with tobacco tissue cultures. Physiol. Plant 15, 473-497. doi: 10.1111/j.1399-3054.1962.tb08052.x

Petersen, J., Eriksson, S. K., Harryson, P., Pierog, S., Colby, T., Bartels, D., et al. (2012). The lysine-rich motif of intrinsically disordered stress protein CDeT11-24 from Craterostigma plantagineum is responsible for phosphatidic acid binding and protection of enzymes from damaging effects caused by desiccation. J. Exp. Bot. 63, 4919-4929. doi: 10.1093/jxb/ers173

Reyes, J. L., Campos, F., Wei, H., Arora, R., Yang, Y., Karlson, D., et al. (2008). Functional dissection of hydrophilins during in vitro freeze protection. Plant Cell Environ. 31, 1781-1790. doi: 10.1111/j.1365-3040.2008.01879.x
Rorat, T. (2006). Plant dehydrins-tissue location, structure and function. Cell Mol. Biol. Lett. 11, 536-556. doi: 10.2478/s11658-006-0044-0

Salleh, F. M., Evans, K., Goodall, B., Machin, H., Mowla, S. B., Mur, L. A. J., et al. (2012). A novel function for a redox-related LEA protein (SAG21/AtLEA5) in root development and biotic stress responses. Plant Cell Environ. 35, 418-429. doi: 10.1111/j.1365-3040.2011.02394.x

Tunnacliffe, A., and Wise, M. J. (2007). The continuing conundrum of LEA proteins. Naturwissenschaften 94, 791-812. doi: 10.1007/s00114-007-0254-y

Wang, L., Liu, Y., Cai, G. H., Jiang, S. S., Pan, J. W., and Li, D. Q. (2014). Ectopic expression of ZmSIMK1 leads to improved drought tolerance and activation of systematic acquired resistance in transgenic tobacco. J. Biotechnol. 172, 18-29. doi: 10.1016/j.jbiotec.2013.11.006

Yang, W., Zhang, L., Lv, H., Li, H., Zhang, Y., Xu, Y., et al. (2015). The K-segments of wheat dehydrin WZY2 are essential for its protective functions under temperature stress. Front. Plant Sci. 6:406. doi: 10.3389/fpls.2015.00406

Yu, J. N., Zhang, J. S., Shan, L., and Chen, S. Y. (2005). Two new group 3 LEA genes of wheat and their functional analysis in yeast. J. Integr. Plant Biol. 47, 1372-1381. doi: 10.1111/j.1744-7909.2005.00126.x

Zhang, L., Ohta, A., Takagi, M., and Imai, R. (2000). Expression of plant group 2 and group 3 lea genes in Saccharomyces cerevisiae revealed functional divergence among LEA proteins. J. Biochem. 127, 611-616. doi: 10.1093/oxfordjournals.jbchem.a022648

Conflict of Interest Statement: The authors declare that the research was conducted in the absence of any commercial or financial relationships that could be construed as a potential conflict of interest.

Copyright (c) 2016 Liu, Liang, Sun, Yang and Li. This is an open-access article distributed under the terms of the Creative Commons Attribution License (CC BY). The use, distribution or reproduction in other forums is permitted, provided the original author(s) or licensor are credited and that the original publication in this journal is cited, in accordance with accepted academic practice. No use, distribution or reproduction is permitted which does not comply with these terms. 